Kansas State University Libraries

New Prairie Press

\title{
COMPARING MEASURING METHODS USING THE SENSITIVITY RATIO: AN APPLICATION TO RESISTANCE SCREENING IN SOYBEANS
}

\author{
Gabotepele Madisa \\ Kent M. Eskridge \\ Kris S. Powers \\ James R. Steadman \\ Rebecca Higgins
}

See next page for additional authors

Follow this and additional works at: https://newprairiepress.org/agstatconference

Part of the Agriculture Commons, and the Applied Statistics Commons (c) (1) $(9)$

This work is licensed under a Creative Commons Attribution-Noncommercial-No Derivative Works 4.0 License.

\section{Recommended Citation}

Madisa, Gabotepele; Eskridge, Kent M.; Powers, Kris S.; Steadman, James R.; Higgins, Rebecca; and Bellows, Connie (2003). "COMPARING MEASURING METHODS USING THE SENSITIVITY RATIO: AN APPLICATION TO RESISTANCE SCREENING IN SOYBEANS," Conference on Applied Statistics in Agriculture. https://doi.org/10.4148/2475-7772.1189

This is brought to you for free and open access by the Conferences at New Prairie Press. It has been accepted for inclusion in Conference on Applied Statistics in Agriculture by an authorized administrator of New Prairie Press. For more information, please contact cads@k-state.edu. 
Author Information

Gabotepele Madisa, Kent M. Eskridge, Kris S. Powers, James R. Steadman, Rebecca Higgins, and Connie Bellows

This is available at New Prairie Press: https://newprairiepress.org/agstatconference/2003/proceedings/20 


\title{
Comparing measuring methods using the sensitivity ratio: An application to resistance screening in soybeans
}

\author{
Gabotepele Madisa ${ }^{1}$, Kent M. Eskridge ${ }^{1}$, Kris S. Powers ${ }^{2}$, James R. Steadman², \\ Rebecca Higgins ${ }^{2}$ and Connie Bellows ${ }^{2}$, \\ 1. Department of Biometry, University of Nebraska - Lincoln \\ 2. Department of Plant Pathology, University of Nebraska - Lincoln
}

\begin{abstract}
When there are several methods of measuring a physical or chemical property, it is necessary to determine which method is best. If both methods are measured on the same scale, the most precise method will be preferred. However, often the methods have different scales. The sensitivity ratio allows for explicit comparison of methods with different scales. We use the sensitivity ratio to compare soybean resistance screening methods to evaluate the resistance of soybean varieties to Sclerotinia sclerotiorum. When compared to the root mean square error or the coefficient of variation, the sensitivity ratio can order methods differently both when the methods are measured on different scales and on the same scale. Our results cast doubt upon using standard precision statistics such as the root mean square error or the coefficient of variation to compare measuring methods and we suggest that the sensitivity ratio should be used instead.
\end{abstract}




\section{INTRODUCTION}

In many scientific investigations, there are often several different ways of measuring the same property. For example, the amount of a particular element in a chemical compound can be measured with a gas chromatograph or with High Performance Liquid Chromatography (HPLC). For a biological example, resistance to white mold (Sclerotinia Sclerotiorum) in soybeans can be measured with either the cut stem method or with the detached leaf method (Kull et al, 2003). When there are several different methods of measuring the same property, it is important to determine which method is best in terms of technical merit, that is, ignoring the differences in costs. A reasonable way to proceed would be to compare the inherent measurement variability of each of the methods using variances or standard deviations. However, different measuring methods will often have different scales of measurement, thus invalidating the direct comparison of standard deviations. To account for different scales, the coefficient of variation (CV) has often been used to compare the technical merit of different measurement methods, however, the $\mathrm{CV}$ is not valid for comparing methods if one scale is not a direct or inverse proportion of the other. As an example of a problem with the $\mathrm{CV}$, assume the mean and standard deviation of temperature for a calendar day at a particular location is $\mu_{\mathrm{F}}=50 \mathrm{~F}^{\circ}$ and $\sigma_{\mathrm{F}}=10 \mathrm{~F}^{\circ}$ and $\mathrm{so} \mu_{\mathrm{C}}=10 \mathrm{C}^{\circ}$ and $\sigma_{C}=5.6 C^{\circ}$. Using these values results in the $C V_{F}=20 \%$ while the $C V_{C}=56 \%$. Choosing to measure temperature in $F^{\circ}$ instead of $C^{\circ}$ because the $C V_{F}<C V_{C}$ is silly since the only difference is the temperature scale. The problem is that the Fahrenheit degrees is not proportional to centigrade degrees. More generally, different measurement devices often are measured on totally different scales where the relationship between the scales is unknown. Consequently any statistical approach of comparing measuring methods should be unaffected by the scales of the different methods. Mandel (1964) presented the sensitivity ratio (SR) as a scale independent approach for comparing measurement methods for technical merit. Surprisingly, the sensitivity ratio has not been widely used for comparing measuring methods and has 
only been used in one biological application (Kull et al., 2003). The objectives of this paper are to describe the concept of the sensitivity ratio and to apply the sensitivity ratio to two resistance screening methods of soybeans to white mold (Sclerotinia Sclerotiorum) in order to determine which method is best in terms of technical merit.

\section{MATERIALS AND METHODS}

Sensitivity Ratio

Assume two methods $\mathrm{M}$ and $\mathrm{N}$ are to be compared regarding their technical merit in their ability to measure a particular property $Q$. To develop the sensitivity ratio, assume method $M$ is used to estimate property $Q$ where $M$ is a function of $Q$, $M=f(Q)$. Assuming $f^{-1}\left(\right.$.) exists, $Q=f^{-1}(M)$, and using the delta rule, it is possible to show that the variance of $Q$ is

$$
\sigma_{Q(M)}^{2}=\left[\mathrm{df}^{-1} / \mathrm{dM}\right]^{2} \sigma^{2} \mathrm{M}
$$

where $\sigma^{2}{ }_{M}$ is the measurement error variance of $M$.

The technical merit (or sensitivity) of method $M$ in measuring $Q$ is defined as $1 / \sigma_{Q(M)}=$ $(d f / d Q) / \sigma_{M}$ since $d f^{-1} / d M=1 /(d f / d Q)$. Note that if $Q=f(Q)$, that is the method actually measures the property $Q$, the technical merit is simply the inverse of the standard deviation $\sigma_{\mathrm{M}}$. Similar results will hold for method $N$ used to measure $Q$ where $N=$ $g(Q)$. Using results from calculus, it can be shown that the sensitivity ratio for the two methods $\mathrm{M}$ and $\mathrm{N}$ is the ratio of the technical merits:

$$
\operatorname{SR}(M / N)=|d M / d N| /\left(\sigma_{M} / \sigma_{N}\right)
$$

where the relationship between the methods is $d M / d N$. If $S R(M / N)>1$ the technical merit of $M$ is superior to $N$, if $S R(M / N)<1$ the technical merit of $N$ is superior and the methods are equal if $S R(M / N)=1$. 
All terms of the sensitivity ratio in [1] may be determined experimentally. Both $\sigma_{M}$ and $\sigma_{N}$ can be estimated using appropriate error variances from designed experiments while $\mathrm{dM} / \mathrm{dN}$ can be estimated from a smooth curve fit through the $\mathrm{M}$ and $\mathrm{N}$ points on a scattergram. If the curve is a straight line, $\mathrm{dM} / \mathrm{dN}$ is the slope from a simple linear regression of $\mathrm{M}$ regressed on $\mathrm{N}$.

Conceptually, the sensitivity ratio is simply the ratio of the inverses of the standard deviations of the methods. However, since the sensitivity ratio explicitly incorporates the functional relationship between each method and $Q$ it is possible to show that the sensitivity ratio is not changed by any transformation of scale. Following Mandel (1964), let $M^{*}$ be a transformation of $M$, ie $M^{*}=h(M)$. Then by definition,

$$
\operatorname{SR}\left(M^{*} / N\right)=\left|d M^{*} / d N\right| /\left(\sigma_{M^{*}} / \sigma_{N}\right)
$$

Also,

$$
\mathrm{dM}^{*} / \mathrm{dN}=\mathrm{dh}(\mathrm{M}) / \mathrm{dN}=(\mathrm{dh}(\mathrm{M}) / \mathrm{dM})(\mathrm{dM} / \mathrm{dN})
$$

and by using the delta rule,

$$
\sigma_{\mathrm{Q}\left(\mathrm{M}^{*}\right)}=|\mathrm{dh} / \mathrm{dM}| \sigma_{\mathrm{M}^{*}}
$$

Now by substituting [3] and [4] into [2], it can be shown that

$$
\operatorname{SR}\left(M^{*} / N\right)=|d M / d N| /\left(\sigma_{M} / \sigma_{N}\right)
$$

Thus, the sensitivity ratio of $M^{*}$ to $N$ is the same as the sensitivity ratio of $M$ to $N$ and so the SR is invariant to scale transformation. Such invariance is a useful property of the sensitivity ratio which no other statistical quantity used for comparing measuring methods has, as far as we know.

An important question is if $S R=1$, that is the two methods are equal in terms of their technical merits. Using experimental data, it is possible to test this hypothesis making use of the F-test (Mandel and Stiehler, 1954). To see this, assume the hypothesis of interest is $H_{0}: S R \geq 1$ vs. $H_{a}: S R<1$. Since $F=\left(s_{M}{ }^{2} / \sigma_{M}{ }^{2}\right) /\left(s_{N}{ }^{2} /\right.$ $\left.\sigma_{N}^{2}\right) \sim F(v 1, v 2)$, by rearranging this relationship we have $\left(\sigma_{M} / \sigma_{N}\right)=(1 / \sqrt{ } F)\left(s_{M} /\right.$ 
$\left.s_{N}\right)$ and so $|d M / d N| /\left(\sigma_{M} / \sigma_{N}\right)=(\sqrt{ }) \mid d M / d N / /\left(s_{M} / s_{N}\right)$. So a reasonable test of $H_{0}$ is to reject $\mathrm{H}_{0}$ if $(\sqrt{ } \mathrm{F}) \overline{S R}<1$ where $\overline{S R}=|\mathrm{dM} / \mathrm{dN}| /\left(\mathrm{s}_{\mathrm{M}} / \mathrm{s}_{\mathrm{N}}\right)$.

Application of the sensitivity ratio to resistance screening in soybeans

Data from soybean white mold (Sclerotinia Sclerotiorum) resistance screening experiments were used to compare methods of evaluating resistance. Three soybean cultivars (Williams82, Bell and NKS19-90) were selected for a range of field reactions to white mold (Wegulo et al 1998). The three cultivars were used in the experiment together with two isolates of white mold, 143 and 279, and this gave rise to six (3X2) treatment combinations of cultivars and isolates of Sclerotinia Sclerotiorum. The two isolates were selected from the University of Nebraska Sclerotinia Sclerotiorum collection and based on previously determined aggressiveness reactions on soybean. Four seeds of each cultivar were planted in 6 inch pots and the seedlings were later thinned to two plants per pot. The pots were arranged on a bench as fifteen incomplete blocks of four pots each with ten pots per treatment (Cochran and Cox, 1957, plan 11.6 page 472). The experiment was run twice where a run was made up of the fifteen incomplete blocks. The data from this experiment was used to compare the cut stem method with the detached leaf test, and digital measurements with hand measurements for the detached leaf test.

Two white mold resistance screening methods were used to evaluate disease reaction of the plants: the detached leaf test (DLT) and the cut stem method (CSM).

Detached leaf test (DLT). On the $28^{\text {th }}$ day, the youngest fuly expanded trifoliate of one plant from each pot was cut with a pair of scissors and transported to plant pathology laboratory in plastic bags containing water. In the lab, the four trifoliates were labeled and placed in aluminum turkey roasting pans which served as incomplete blocks in accordance to how they were randomized in the green house. Four folded paper towels were placed at the bottom of each pan and four glass Petri 
dishes were placed upside down in each pan on paper towels. Orchid tubes were filled with tap water, capped and placed in pans with one tube placed under each Petri dish. The petiole (stem) of each trifoliate was pushed through the orchid tube cap until the cut end reached the water. The middle leaf of each trifoliate was placed on top of the petri dish and inoculated with an isolate. The plug was placed on one side of the leaf between the main leaf vein and the leaf edge and was gently pressed to ensure a good contact with the leaf surface. The pans containing the inoculated leaves were wrapped with a plastic wrap to maintain humidity. The pans were then left on the bench for 48 hours at $20 \pm 2^{\circ} \mathrm{C}$. After 48 hours the lesions were measured digitally (using a digital camera).

Another DLT experiment was conducted to compare digital lesion size measurements with hand measurements (in centimeters) obtained using a ruler where the mean of two lesion diameters taken in two perpendicular directions was used to compute the area of a circle. This experiment was conducted as the previously described experiment except there were 56 soybean lines and one isolate. In addition, there were four replicates where four lines were assigned to each pan (incomplete block) using an alpha lattice design giving a total of 14 incomplete blocks per replicate.

Cut stem method (CSM). After cutting a trifoliate from one of the plants in the pot, that plant was removed leaving one plant for the cut stem method. After five weeks of growth, the plant was removed from the pot. Main stems of the 5-week old plants were horizontally cut with a sterile razor blade $0.5 \mathrm{~cm}$ above the fourth or fifth node. A white mold plug was immediately placed on the stem and then the inoculated plants were incubated in a mist chamber with humidity maintained over $80 \%$. The temperature was maintained at $20 \pm 1^{\circ} \mathrm{C}$ and covered with a black mesh cloth to reduce $80 \%$ of the light. After 24 hours of incubation, the plants were transferred to an adjacent room where the temperature was maintained at $25 \pm 1^{\circ} \mathrm{C}$ and the disease symptoms were allowed to develop. Disease development was observed and lesion lengths $(\mathrm{cm})$ on the main stems were manually measured 14 days after inoculation. 
Statistical analyses were performed to compare two different pairs of methods: (1) CSM vs DLT digital lesion measurements (Table 1) and (2) DLT digital vs DLT hand measurements (data not shown). The sensitivity ratio was estimated using the root mean square error (RMSE) from the analysis of variance of each method ( $M=D L T$ digital vs $\mathrm{N}=\mathrm{CSM}$ or $\mathrm{M}=\mathrm{DLT}$ hand vs $\mathrm{N}=\mathrm{DLT}$ digital) to estimate $\sigma_{\mathrm{M}}$ and $\sigma_{\mathrm{N}}$ and using the slope of a simple linear regression of $M$ regressed on $N$ to estimate $d M / d N$. The root mean square error (RMSE), coefficient of variation (CV), slope, correlation between $\mathrm{M}$ and $\mathrm{N}$ and the SR were used to compare the methods. The F-test was used to test the hypothesis $\mathrm{H}_{0}$ : $\mathrm{SR} \geq 1$.

\section{RESULTS AND DISCUSSION}

When comparing the technical merits of the DLT and the CSM screening methods using the RMSE, the DLT was better than the CSM (1.79 vs 2.48: Table 2). However, RMSE is scale dependent and therefore does not present a valid comparison of technical merit of the two methods. The coefficient of variation (CV) indicated that CSM was somewhat better ( $24 \%$ vs $17 \%$ ), however, as with RMSE, the coefficient of variation is scale dependent. The Pearson correlation coefficient between adjusted treatment means of DLT and CSM was 0.691 which indicated a reasonable linear relationship between the two methods. The slope of the simple linear regression of adjusted treatment means of the DLT regressed on the CSM adjusted treatment means showed a positive linear relationship between the CSM and $\mathrm{DLT}$. Using the slope to estimate $\mathrm{dM} / \mathrm{dN}$, the sensitivity ratio of the DLT with respect to the CSM was less than one (0.318: Table 2), which meant that the CSM was substantially superior to the DLT with the CSM being roughly 9 times better than the DLT. That is, to obtain a SR of 1 , it would take approximately $9\left((1 / .318)^{2}\right)$ times as many samples of the DLT as compared to the CSM (Mandel, 1964). In addition, using the F-test as described above, the SR (DLT/CSM) was significantly less than 
one at $5 \%$ level, therefore it may be concluded that CSM was more sensitive than DLT.

In comparison of the DLT hand vs digital measurements, the RMSE ordered the measurement methods as (1) hand method and (2) digital method (Table 2). The CV indicated that the hand method was essentially the same as digital although there was a very slight advantage to hand (34\% vs 35\%: Table 2). The two methods were reasonably correlated (0.634: Table 2$)$ and had a positive slope (0.455: Table 2$)$. The SR (hand/digital) was 0.678 (Table 2) which meant that digital was approximately 2 times better than the hand measurement method. The SR (hand/digital) was significantly less than one at $5 \%$ level, therefore digital was more sensitive than the hand method.

The above results show that the RMSE and the CV can order methods differently from the sensitivity ratio. One reason for this difference in ranking is apparently due to the failure of these methods to be scale independent. However, even if the methods are based on the same measurement scale, as with the DLT digital vs hand comparison (both were measured in $\mathrm{cm}^{2}$ ), RMSE and CV can give orderings different from SR. The reason for this difference, when the methods are on the same scale, is not clear.

There are several important assumptions that should be kept in mind when using the sensitivity ratio. We assumed a linear relationship between the two methods ( $M$ and $\mathrm{N}$ ) being compared and that $\mathrm{dM} / \mathrm{dN}$ was adequately estimated by the slope of a simple linear regression of the means of $M$ regressed on the means of $N$. If the methods are not related or poorly related, the SR is of questionable values. Also, if the methods are related curvilinearly, then SR will depend on the slope (or tangent) at a particular point. However, curvilinearity should not be a problem as long as the region can be identified of $\mathrm{M}$ and $\mathrm{N}$ where $\mathrm{SR}$ is relevant. In addition, the standard deviations $\left(\sigma_{M}\right.$ and $\left.\sigma_{N}\right)$ can depend on the sizes of $M$ and $N$ but this again should not be a problem as long as the relevant region of $\mathrm{M}$ and $\mathrm{N}$ can be identified. Another more difficult problem is that sensitivity ratios are based on the assumption of a near 
perfect regression relationship between $\mathrm{M}$ and $\mathrm{N}$ which does not hold with these data. Often a poor regression relationship will result in biased estimates of the sensitivity ratio (Mandel, 1964). Using a method to correct for bias as suggested in Snedecor and Cochran (1967), we estimated that the slopes were biased downward by approximately $10 \%$. Adjusting the sensitivity ratios upward using this $10 \%$ bias of the slopes increased the SR to 0.35 for DLT vs CSM and to 0.75 for DLT hand vs digital. Even adjusting for this bias, the same conclusions hold.

\section{SUMMARY}

The sensitivity ratio is a scale independent statistical quantity that is useful for comparing the technical merit of different methods for measuring a physical or chemical property. Commonly used measures such as the root mean square error and coefficient of variation are not scale independent and thus should be avoided for comparing measuring methods. We used the RMSE, CV and SR to compare several methods of measuring soybean resistance to white mold. When compared to the RMSE or the CV, the SR ordered methods differently and thus casts doubt upon using these standard precision statistics to compare different measuring methods. Consequently, we suggest that the sensitivity ratio should be used whenever it is necessary to compare two measuring methods.

\section{REFERENCES}

Kull, L. S., T.D. Vuong, K.S. Powers, K. M. Eskridge, G.L. Hartman, and J.R. Steadman. 2003. Evaluation of Three Resistance Screening Methods Using Six Sclerotinia sclerotiorum Isolates and Three Entries of Each Soybean and Dry Bean. Plant Disease. In press.

Mandel, J., and R.D Stiehler. 1954. A Criterion for the Comparison of Methods of Test. Research Paper 2527 Journal of Research of the National Bureau Standards. 53(3):155-159. 
Mandel, J. 1964. The statistical analysis of experimental data. Wiley, New York

Snedecor, G. W. and W. G. Cochran. 1967. Statistical Methods. $6^{\text {th }}$ ed. lowa State Univ. Press. Ames, IA.

Wegulo, S. N., Yang, X. B., and Martinson, C. A. 1998. Soybean cultivar responses to Sclerotinia sclerotiorum in field and controlled environmental studies. Plant Disease. 82:1264-1270.

Table 1. Means of $\mathrm{CSM}^{+}$and $\mathrm{DLT}^{\#}$ methods for evaluating resistance to Sclerotinia sclerotiorum on soybeans for three soybean cultivars and two isolates of Sclerotinia sclerotiorum .

\begin{tabular}{|c|c|c|c|}
\hline Cultivar & Isolate & CSM $^{+}$ & DLT $^{\#}$ \\
\hline Bell & 143 & 13.65 & 7.36 \\
\hline Bell & 279 & 13.04 & 8.07 \\
\hline NKS1990 & 143 & 11.97 & 6.77 \\
\hline NKS1990 & 279 & 13.42 & 6.42 \\
\hline Williams 82 & 143 & 16.80 & 7.72 \\
\hline Williams 82 & 279 & 18.12 & 8.56 \\
\hline Std error & & 0.61 & 0.43 \\
\hline
\end{tabular}

+ Cut Stem Method - lesion lengths in $\mathrm{cm}$.

\# Detached Leaf Method - digitally measured area in $\mathrm{cm}^{2}$.

* Average standard error 
Table 2. Root mean square error (RMSE), coefficient of variation (CV), correlation between methods (Corr), slope of simple linear regression (slope) and sensitivity ratio (SR) for methods of evaluating resistance to Sclerotinia sclerotiorum on soybeans

\begin{tabular}{|c|c|c|c|c|c|}
\hline \multicolumn{7}{|c|}{ Detached Leaf vs Cut Stem } \\
\hline METHOD & RMSE & CV & Corr & Slope & SR \\
\hline DLT & 1.792 & $24 \%$ & 0.691 & 0.230 & 0.318 \\
\hline CSM & 2.477 & $17 \%$ & & & \\
\hline \multicolumn{7}{|c|}{ Detached Leaf Hand vs Digital } \\
\hline Hand & 1.373 & $34 \%$ & 0.634 & 0.455 & 0.678 \\
\hline Digital & 2.045 & $35 \%$ & & & \\
\hline
\end{tabular}

